\title{
SPECTRAL CONDITIONS FOR ALMOST COMPOSITION OPERATORS BETWEEN ALGEBRAS OF FUNCTIONS
}

\author{
T. TONEV
}

(Communicated by Richard Rochberg)

\begin{abstract}
In this article we develop a unifying theory of many years of work by a number of researchers. Especially, we establish general sufficient conditions for maps between algebras of bounded continuous functions on locally compact Hausdorff spaces to be almost composition or almost weighted composition operators, which extend the main results of many previous works on this subject. The following are some typical results. Let $T: A \rightarrow B$ be a surjective map between two function algebras on locally compact Hausdorff spaces $X$ and $Y$ with Choquet boundaries $\delta A \subset X$ and $\delta B \subset Y$. If $\|T f T g\|=\|f g\|$ and there is an $\varepsilon, 0 \leq \varepsilon<2 / 3$, so that the peripheral spectrum $\sigma_{\pi}(T f T g)$ is contained in an $\varepsilon\|f g\|$-neighborhood of $\sigma_{\pi}(f g)$ for all $f \in A$ and all $g \in A$ with $\|g\|=1$, then there is a continuous function $\alpha: \delta B \rightarrow\{ \pm 1\}$ and a homeomorphism $\psi: \delta B \rightarrow \delta A$ so that $|(T f)(y)-\alpha(y) f(\psi(y))| \leq 2 \varepsilon|f(\psi(y))|$ for each $f \in A$ and every $y \in \delta B$. Therefore, $T$ is an almost weighted composition operator on $\delta B$. If $\|T f T g\|=\|f g\|$, there are $\varepsilon, 0 \leq \varepsilon<1$, and $\eta, 0 \leq \eta<1$, so that $d\left(\sigma_{\pi}(T f T g), \sigma_{\pi}(f g)\right) \leq \varepsilon\|f g\|$, while $\sigma_{\pi}(T f)$ is contained in an $\eta$ neighborhood of $\sigma_{\pi}(f)$ for all $f \in A$ and all $g \in A$ with $\|g\|=1$. Then $|(T f)(y)-f(\psi(y))| \leq(\varepsilon+\eta)|f(\psi(y))|$ for each $y \in \delta B$ and every $f \in A$; i.e. $T$ is an almost composition operator on $\delta B$. If $\sigma_{\pi}(T f T g) \subset \sigma_{\pi}(f g)$ (or $\left.\sigma_{\pi}(f g) \subset \sigma_{\pi}(T f T g)\right)$, and $d\left(\sigma_{\pi}(T g), \sigma_{\pi}(g)\right) \leq \eta$ for some $\eta, 0 \leq \eta<1$, for all $f \in A$ and all $g \in A$ with $\|g\|=1$, then $(T f)(y)=f(\psi(y))$ for each $f \in A$ and every $y \in \delta B$. Consequently, $T$ is a composition operator on $\delta B$ and, therefore, an algebra isomorphism.
\end{abstract}

\section{Preliminaries}

The search of spectral conditions for mappings $T: A \rightarrow B$ between algebras of functions to be composition or weighted composition operators is a dynamic area of research. In particular, the peripherally multiplicative conditions

$$
\sigma_{\pi}(T f T g)=\sigma_{\pi}(f g) \text { and } \sigma_{\pi}(T f T g) \cap \sigma_{\pi}(f g) \neq \varnothing
$$

and their variations, where $\sigma_{\pi}(f)$ is the peripheral spectrum of $f \in A$, have attracted considerable interest (cf. [1-3, 8, 10]).

Recall that an algebra $A$ of bounded continuous functions on a locally compact Hausdorff set $X$ is called a function algebra if $A$ is closed under the uniform norm $\|f\|=\sup _{x \in X}|f(x)|$ and separates strongly the points of $X$, i.e. for every $x \in X$

Received by the editors April 30, 2012 and, in revised form, August 31, 2012.

2010 Mathematics Subject Classification. Primary 46J10.

Key words and phrases. Function algebra, uniform algebra, peripheral spectrum, composition operator, almost composition operator, almost weighted composition operator, algebra isomorphism, Choquet boundary.

This research was partially supported by grant No. 209762 from the Simons Foundation. 
there is an $f \in A$ with $f(x) \neq 0$, and for every $x, y \in X$ there is an $f \in A$ with $f(x) \neq f(y)$. If, in addition, $A$ contains the constant functions on $X$, then it is called a uniform algebra. We assume that the uniform closures $[A]$ of all algebras $A$ in this paper are function algebras and also that their underlying spaces $X$ contain their Choquet (or strong) boundaries $\delta A$. Under these conditions, $\|f\|=$ $\sup _{x \in X}|f(x)|=\max _{x \in X}|f(x)|=\max _{x \in \delta A}|f(x)|$.

Let $A$ be an algebra of bounded continuous functions on a locally compact Hausdorff space $X$. Recall that the closure of the Choquet boundary $\delta A=\delta[A]$ of $A$ in the Gelfand topology of the maximal ideal space of the uniform closure $[A]$ of $A$ is the Shilov boundary $\partial A=\partial[A]$ of $A$. A function $h \in A$ is called a peaking function of $A$, provided $\|h\|=1$ and $|h(x)|<1$ whenever $h(x) \neq 1$, and a peak function of $A$ if $h$ is a peaking function of $A$ that takes its maximum modulus at a single point. We denote by $\mathcal{P}(A)$ [resp. $\mathcal{P}^{0}(A)$ ] the set of all peaking [resp. peak] functions of $A$, and by $\mathcal{P}_{x_{0}}(A)=\left\{h \in \mathcal{P}(A): h\left(x_{0}\right)=1\right\}\left[\right.$ resp. $\left.\mathcal{P}_{x_{0}}^{0}(A)=\left\{h \in \mathcal{P}^{0}(A): h\left(x_{0}\right)=1\right\}\right]$ those that peak at $x_{0}$. The set $\sigma_{\pi}(f)$ of range values with maximum modulus of an $f \in A$, i.e. $\sigma_{\pi}(f)=\{f(x):|f(x)|=\|f\|, x \in X\}$, is called the peripheral spectrum of $f$. Clearly, $\sigma_{\pi}(f) \subset\{z \in \mathbb{C}:|z|=\|f\|\}$. Note that the peripheral spectrum of an element $f$ of a function algebra $A$ without unit coincides with the peripheral spectrum of $f$ as an element of the unification $A+\mathbb{C}$ of $A$, since the spectrum of $f$ as an element of $A+\mathbb{C}$ might differ from the spectrum of $f$ as an element of $A$ only by $\{0\}$. The set $E(f)=f^{-1}\left\{\sigma_{\pi}(f)\right\}=\{x \in X:|f(x)|=\|f\|\}$ is called the maximizing set of $f \in A$. Clearly, $f(x) \in \sigma_{\pi}(f)$ if and only if $x \in E(f)$. A set $E \subset X$ is a peak set of $A$ if $E=E(h)=h^{-1}\{1\}$ for some peaking function $h \in \mathcal{P}(A)$. Singleton peak sets are called peak points. A point $x_{0} \in X$ is a $p$-point, or a generalized peak point, if it is an intersection of peak sets. The set of all $p$-points [resp. peak points] of $A$ is denoted by $p(A)$ [resp. $\left.p_{0}(A)\right]$. In general, $p_{0}(A) \subsetneq p(A) \subsetneq \delta A$, and even $p(A)$ can be empty. However, if $A$ is a function algebra, then necessarily, $p(A)=\delta A \neq \varnothing$. Moreover, if $A$ is a function algebra on a metric space or the algebra of all Lipschitz functions on a metric space, then $p_{0}(A)=p(A)=\delta A$ (cf. [7]). Given a set $S \subset \mathbb{C}$ and an $r>0, \mathcal{O}_{r}(S)$ will denote the closed $r$-neighborhood of $S$ in $\mathbb{C}$, i.e. $\mathcal{O}_{r}(S)=\{z \in \mathbb{C}: d(z, S) \leq r\}$, where $d(u, v)=|u-v|, u, v \in \mathbb{C}$, is the Euclidean distance in $\mathbb{C}$.

Given two algebras of bounded continuous functions, $A \subset C(X)$ and $B \subset C(Y)$, and a continuous map $\psi: Y \rightarrow X$, a mapping $T: A \rightarrow B$ is said to be: a composition operator on $Y$, provided $(T f)(y)=f(\psi(y))$; a composition operator in modulus on $Y$ if $|(T f)(y)|=|f(\psi(y))|$; a weighted composition operator on $Y$, provided there is a non-vanishing function $\alpha \in C(Y)$ so that $(T f)(y)=\alpha(y) f(\psi(y))$; an almost composition operator on $Y$ if there is an $\varepsilon \geq 0$ so that $|(T f)(y)-f(\psi(y))| \leq \varepsilon|f(\psi(y))|$; an almost weighted composition operator on $Y$, provided there is a non-vanishing function $\alpha \in C(Y)$ so that $|(T f)(y)-\alpha(y) f(\psi(y))| \leq \varepsilon|f(\psi(y))|$ for each $f \in A$ and every $y \in Y$. In order for a map $T=\alpha(f \circ \psi)$ to be a weighted composition operator, the function $\alpha$ must depend solely on $x$ and not on $f$. If the mappings $\psi: Y \rightarrow X$ from above are homeomorphisms, then the corresponding composition operators on $Y$ are isomorphisms between algebras, while almost composition operators on $Y$ are almost isomorphisms between algebras. The latter maps were studied by Jarosz [4] in a different context.

In this paper we develop a unifying theory of many years of work by several researchers. Especially, we generalize the peripheral multiplicative properties in (1) 
and establish general sufficient conditions, such as $\sigma_{\pi}(T f T g) \subset \mathcal{O}_{\varepsilon\|f g\|}\left(\sigma_{\pi}(f g)\right)$ and $d\left(\sigma_{\pi}(T f T g), \sigma_{\pi}(f g)\right) \leq \varepsilon\|f g\|$, for surjective mappings between algebras of bounded continuous functions on locally compact Hausdorff spaces to be almost composition or almost weighted composition operators which extend and generalize several of the main results in the area. Here is a short preview of the main results.

Theorem 1. Let $A \subset C(X)$ and $B \subset C(Y)$ be function algebras with $\delta A \subset X$ and $\delta B \subset Y$. If a surjective map $T: A \rightarrow B$ satisfies

(i) $\sigma_{\pi}(T f T g) \subset \mathcal{O}_{\varepsilon\|f g\|}\left(\sigma_{\pi}(f g)\right)$ for some $\varepsilon, 0 \leq \varepsilon<2 / 3$ and

(ii) $\|T f T g\|=\|f g\|$

for all $f \in A$ and all $g \in A$ with $\|g\|=1$, then there is a homeomorphism $\psi: \delta B \rightarrow$ $\delta A$ and a continuous function $\alpha: \delta B \rightarrow\{ \pm 1\}$ such that

$$
|(T f)(y)-\alpha(y) f(\psi(y))| \leq 2 \varepsilon|f(\psi(y))|
$$

for each $f \in A$ and every $y \in \delta B$. Consequently, $T$ is an almost weighted composition operator on $\delta B$.

This theorem holds also if, instead of $(i), T$ satisfies its symmetric condition $\sigma_{\pi}(f g) \subset \mathcal{O}_{\varepsilon\|f g\|}\left(\sigma_{\pi}(T f T g)\right)$. The main results in [3, 5, 9, 12, are immediate consequences of Theorem 1. If, in addition to conditions $(i)$ and $(i i)$, there is an $\eta, 0 \leq \eta<1$, so that $d\left(\sigma_{\pi}(T g), \sigma_{\pi}(g)\right) \leq \eta$ for all $g \in A$ with $\|g\|=1$, then $|(T f)(y)-f(\psi(y))| \leq 2 \varepsilon|f(\psi(y))|$ for each $f \in A$ and every $y \in \delta B$. Hence $T$ is an almost composition operator on $\delta B$, and therefore is an almost isomorphism between algebras.

Theorem 3. If a surjection $T: A \rightarrow B$ between function algebras satisfies

(i) $\sigma_{\pi}(T f T g) \subset \sigma_{\pi}(f g)\left(\right.$ or, $\left.\sigma_{\pi}(f g) \subset \sigma_{\pi}(T f T g)\right)$ and

(ii) $d\left(\sigma_{\pi}(T g), \sigma_{\pi}(g)\right) \leq \eta$ for some $\eta, 0 \leq \eta<1$,

for all $f \in A$ and all $g \in A$ with $\|g\|=1$, then there is a homeomorphism $\psi: \delta B \rightarrow$ $\delta A$ so that

$$
(T f)(y)=f(\psi(y))
$$

for each $f \in A$ and every $y \in \delta B$. Consequently, $T$ is a composition operator on $\delta B$ and, therefore, an algebra isomorphism.

This theorem generalizes and extends the main results in [5] and [9].

Theorem 4. Let $A \subset C(X), B \subset C(Y)$ be function algebras with $\delta A \subset X$ and $\delta B \subset Y$. If a surjective map $T: A \rightarrow B$ satisfies

(i) $d\left(\sigma_{\pi}(T f T g), \sigma_{\pi}(f g)\right) \leq \varepsilon\|f g\|$ for some $\varepsilon, 0 \leq \varepsilon<1$,

(ii) $\sigma_{\pi}(T g) \subset \mathcal{O}_{\eta}\left(\sigma_{\pi}(g)\right)\left(\right.$ or $\left.\sigma_{\pi}(g) \subset \mathcal{O}_{\eta}\left(\sigma_{\pi}(T g)\right)\right)$ for some $\eta, 0 \leq \eta<1$, and

(iii) $\|T f T g\|=\|f g\|$

for all $f \in A$ and all $g \in A$ with $\|g\|=1$, then there is a homeomorphism $\psi: \delta B \rightarrow$ $\delta A$ so that

$$
|(T f)(y)-f(\psi(y))| \leq(\varepsilon+\eta)|f(\psi(y))|
$$

for each $f \in A$ and every $y \in \delta B$. Hence, $T$ is an almost composition operator on $\delta B$, and therefore an almost isomorphism between algebras.

The main results in [5,8, 14, are immediate corollaries of this theorem. In some cases condition (ii) in Theorem 4 is unnecessary. 
Theorem 6. Let $A \subset C(X)$ and $B \subset C(Y)$ be dense subalgebras of function algebras with $p(A)=\delta A \subset X$ and $p(B)=\delta B \subset Y$. Assume that for every $f \in A$ and each $x \in \delta A$ with $f(x) \neq 0$ there is a peak function $h \in \mathcal{P}_{x}^{0}(A)$ such that $\sigma_{\pi}(f h)=\{f(x)\}$. If a surjective map $T: A \rightarrow B$ satisfies

(i) $d\left(\sigma_{\pi}(T f T g), \sigma_{\pi}(f g)\right) \leq \varepsilon\|f g\|$ for some $\varepsilon, 0 \leq \varepsilon<2 / 3$ and

(ii) $\|T f T g\|=\|f g\|$

for all $f \in A$ and all $g \in A$ with $\|g\|=1$, then there is a homeomorphism $\psi: \delta B \rightarrow$ $\delta A$ and a continuous function $\alpha: A \rightarrow\{ \pm 1\}$ such that

$$
|(T f)(y)-\alpha(y) f(\psi(y))| \leq 2 \varepsilon|f(\psi(y))|
$$

for each $f \in A$ and every $y \in \delta B$. Therefore, $T$ is an almost weighted composition operator on $\delta B$.

The main results in 6, 7, 13, are direct consequences of Theorem 6 .

\section{THE MAIN THEOREMS}

Let $T: A \rightarrow B$ be a surjective map between two algebras $A$ and $B$ of bounded continuous functions on locally compact Hausdorff spaces $X$ and $Y$. It is known that if $T$ is norm-multiplicative with respect to the uniform norm, i.e. if $\|T f T g\|=\|f g\|$ for all $f, g \in A$, then $T$ is a composition operator in modulus on $\delta B$, i.e. there exists a homeomorphism $\psi: \delta B \rightarrow \delta A$ between the Choquet boundaries so that $|(T f)(y)|=|f(\psi(y))|$ for all $f \in A$ and $y \in \delta B$. This was shown in 3, 8, for uniform algebras, and in [14] for dense subalgebras of function algebras with $p(A)=\delta A \subset X$ and $p(B)=\delta B \subset Y$. The proof given in 14] actually needs the condition $\|T f T g\|=\|f g\|$ for all $f \in A$ and all $g \in A$ with $\|g\|=1$ only. Clearly, every weighted composition operator $T=\alpha(f \circ \psi)$ with $|\alpha| \equiv 1$ is a norm-multiplicative mapping. However, in general, a norm-multiplicative mapping is not obliged to be a weighted composition operator. For instance, if $X$ is a compact Hausdorff space, then the map $S: C(X) \rightarrow C(X)$ defined as $(S f)(x)=f^{2}(x) /|f(x)|=(f /|f|)(x) f(x)$ (and $(S f)(x)=0$ if $\left.f(x)=0\right)$ is normmultiplicative, but not a weighted composition operator since, besides $x$, the function $\alpha=f /|f|$ depends also on $f \in A$.

Lemma 1. Let $A$ and $B$ be algebras of bounded continuous functions on locally compact Hausdorff spaces $X$ and $Y$ with $p(A) \subset X$ and $p(B) \subset Y$, and suppose that $\psi: p(B) \rightarrow p(A)$ is a continuous map. Let $y_{0} \in P(B), f \in A$ is such that $f\left(\psi\left(y_{0}\right)\right) \neq 0$, and suppose that $\sigma_{\pi}(f h)=\left\{f\left(\psi\left(y_{0}\right)\right)\right\}$ for some $h \in \mathcal{P}_{\psi\left(y_{0}\right)}(A)$. If $T: A \rightarrow B$ satisfies $|(T f)(y)|=|f(\psi(y))|$ for all $y \in p(B)$, and if there is an $\varepsilon \geq 0$ such that $\sigma_{\pi}(T f T h) \subset \mathcal{O}_{\varepsilon}\|f h\|\left(\sigma_{\pi}(f h)\right)$, then

$$
\left|(T f T h)\left(y_{0}\right)-f\left(\psi\left(y_{0}\right)\right)\right| \leq \varepsilon\left|f\left(\psi\left(y_{0}\right)\right)\right| .
$$

Proof. Let $y_{0} \in p(B), x_{0}=\psi\left(y_{0}\right) \in p(A)$ and let $f \in A$ be such that $f\left(x_{0}\right) \neq 0$. If $h \in \mathcal{P}_{x_{0}}(A)$ is a peaking function with $\sigma_{\pi}(f h)=\left\{f\left(x_{0}\right)\right\}$, then $x_{0} \in E(f h)$. Therefore, $y_{0} \in E(T f T h)$ since $|(T f T h)(y)|=|(f h)(\psi(y))|$ for all $y \in \delta B$. Hence $(T f T h)\left(y_{0}\right) \in \sigma_{\pi}(T f T h)$. Consequently, $\left|(T f T h)\left(y_{0}\right)-f\left(x_{0}\right)\right| \leq \varepsilon\|f h\|=$ $\varepsilon\left|f\left(x_{0}\right)\right|$, since, by the hypotheses, $\sigma_{\pi}(T f T h) \subset \mathcal{O}_{\varepsilon\|f h\|}\left(\sigma_{\pi}(f h)\right)$.

Lemma 2. Let $A$ and $B$ be algebras of bounded continuous functions on locally compact Hausdorff spaces $X$ and $Y$ with $p(A) \subset X$ and $p(B) \subset Y$, and let $\psi: p(B) \rightarrow$ $p(A)$ be a continuous map. Suppose that $T: A \rightarrow B$ satisfies $|(T h)(y)|=|h(\psi(y))|$ 
for each $y \in p(B)$ and every peaking function $h$ of $A$. If there is an $\varepsilon, 0 \leq \varepsilon<2 / 3$, such that $\sigma_{\pi}\left(T h_{1} T h_{2}\right) \subset\{z \in \mathbb{C}:|z-1| \leq \varepsilon\}=\mathcal{O}_{\varepsilon}(1)$ for any pair of peaking functions $h_{1}, h_{2} \in \mathcal{P}_{x}(A)$ and all $x \in \delta A$, then there is a continuous function $\alpha: p(B) \rightarrow\{ \pm 1\}$ so that

$$
|(T h)(y)-\alpha(y)| \leq \varepsilon
$$

for every $y \in p(B)$ and each $h \in \mathcal{P}_{\psi(y)}(A)$.

Proof. Fix a $p$-point $y_{0}$ of $B$ and let $x_{0}=\psi\left(y_{0}\right) \in p(A)$. Clearly, if $h_{1}$ and $h_{2}$ are peaking functions of $A$ that peak at $x_{0}$, then $\sigma_{\pi}\left(h_{1} h_{2}\right)=\left\{\left(h_{1} h_{2}\right)\left(x_{0}\right)\right\}=\{1\}$. By the hypothesis we have $\left|\left(T h_{1} T h_{2}\right)\left(y_{0}\right)\right|=\left|\left(h_{1} h_{2}\right)\left(x_{0}\right)\right|=1$ and $\left\|T h_{1} T h_{2}\right\|=$ $\left\|h_{1} h_{2}\right\|=1$. Hence $\left(T h_{1} T h_{2}\right)\left(y_{0}\right) \in \sigma_{\pi}\left(T h_{1} T h_{2}\right)$. The condition $\sigma_{\pi}\left(T h_{1} T h_{2}\right) \subset$ $\{z \in \mathbb{C}:|z-1| \leq \varepsilon\}$ implies that the inequality

$$
\left|\left(T h_{1} T h_{2}\right)\left(y_{0}\right)-1\right| \leq \varepsilon
$$

holds for any pair, $h_{1}, h_{2}$, of peaking functions in $\mathcal{P}_{x_{0}}(A)$. We claim that there is a number $\alpha_{h}\left(y_{0}\right)= \pm 1$, depending possibly on $h \in \mathcal{P}_{x_{0}}(A)$ and $y_{0} \in \delta B$, so that

$$
\left|\alpha_{h}\left(y_{0}\right)(T h)\left(y_{0}\right)-1\right| \leq \varepsilon .
$$

Indeed, (4) implies that $\left|(T h)^{2}\left(y_{0}\right)-1\right| \leq \varepsilon<2$ for any $h \in \mathcal{P}_{x_{0}}(A)$, and therefore, $(T h)^{2}\left(y_{0}\right) \neq-1$. Consequently, $(T h)^{2}\left(y_{0}\right)=e^{i A}$ for some real $A$ with $-\pi<$ $A<\pi$. Hence, $(T h)\left(y_{0}\right)=\sqrt{(T h)^{2}\left(y_{0}\right)}= \pm e^{i A / 2}$. If $(T h)\left(y_{0}\right)=e^{i A / 2}$, then $\left|(T h)\left(y_{0}\right)-1\right|=\left|e^{i A / 2}-1\right| \leq\left|e^{i A}-1\right|=\left|(T h)^{2}\left(y_{0}\right)-1\right| \leq \varepsilon$. If $(T h)\left(y_{0}\right)=-e^{i A / 2}$, then $\left|-(T h)\left(y_{0}\right)-1\right|=\left|e^{i A / 2}-1\right| \leq\left|e^{i A}-1\right| \leq \varepsilon$. Since $\varepsilon<2 / 3<\pi / 2$, the numbers $\pm e^{i A / 2}$ cannot be simultaneously $\varepsilon$-close to 1 . Hence there is a unique number $\alpha_{h}\left(y_{0}\right)= \pm 1$ so that $\left|\alpha_{h}\left(y_{0}\right)(T h)\left(y_{0}\right)-1\right|<\varepsilon$, as claimed.

In fact, the number $\alpha_{h}\left(y_{0}\right)= \pm 1$ depends solely on $y_{0} \in \delta B$ and does not depend on the peaking function $h \in \mathcal{P}_{\psi\left(y_{0}\right)}(A)$. Indeed, since $\left|\left(T h_{1}\right)\left(y_{0}\right)\right|=\left|h_{1}\left(\psi\left(y_{0}\right)\right)\right|=1$, by (44) and (5) we deduce:

$$
\begin{gathered}
\left|\left(\alpha_{h_{1}} \alpha_{h_{2}}\right)\left(y_{0}\right)-1\right| \leq\left|\left(\alpha_{h_{1}} \alpha_{h_{2}}\right)\left(y_{0}\right)-\left(\alpha_{h_{1}} T h_{2}\right)\left(y_{0}\right)\right| \\
+\left|\left(\alpha_{h_{1}} T h_{2}\right)\left(y_{0}\right)-\left(T h_{1} T h_{2}\right)\left(y_{0}\right)\right|+\left|\left(T h_{1} T h_{2}\right)\left(y_{0}\right)-1\right| \\
\leq\left|\left(T h_{2}\right)\left(y_{0}\right)-\alpha_{h_{2}}\left(y_{0}\right)\right|+\left|\left(T h_{1}\right)\left(y_{0}\right)-\alpha_{h_{1}}\left(y_{0}\right)\right|+\varepsilon \leq 3 \varepsilon<2 .
\end{gathered}
$$

Therefore, $\left(\alpha_{h_{1}} \alpha_{h_{2}}\right)\left(y_{0}\right)=1$, thus $\alpha_{h_{1}}\left(y_{0}\right)=\alpha_{h_{2}}\left(y_{0}\right)$, since $\alpha_{h_{i}}\left(y_{0}\right)= \pm 1, i=1,2$. Consequently, the number $\alpha_{h}\left(y_{0}\right)$ does not depend on the peaking function $h \in$ $\mathcal{P}_{\psi\left(y_{0}\right)}(A)$, as claimed.

Define a function $\alpha: p(B) \rightarrow\{ \pm 1\}$ by $\alpha(y)=\alpha_{h}(y)$, where $h$ is any peaking function in $A$ that peaks at $\psi(y)$. According to (5), $|(T h)(y)-\alpha(y)|=$ $|\alpha(y)(T h)(y)-1| \leq \varepsilon<1$ for any $y \in p(B)$ and each $h \in \mathcal{P}_{\psi(y)}(A)$. Since $\alpha= \pm 1$ and $T h$ is continuous at $y$, we deduce that $\alpha$ is also continuous at $y$.

Note that Lemma 2 holds not only for the set $\mathcal{P}_{\psi\left(y_{0}\right)}(A)$ of peaking functions, but also for the set $\mathcal{P}_{\psi\left(y_{0}\right)}^{0}(A)$ of peak functions of $A$ that peak at $\psi\left(y_{0}\right)$. In the sequel we make use of the following:

Proposition 1 (The Strong Bishop's Lemma [14]). Let $A$ be a function algebra on $X$ not necessarily with unit. For any $f \in A$ and $x_{0} \in p(A)$ with $f\left(x_{0}\right) \neq 0$, there is a peaking function $h_{0} \in \mathcal{P}_{x_{0}}(A)$ with $E\left(f h_{0}\right)=E\left(h_{0}\right)$ such that $\sigma_{\pi}\left(f h_{0}\right)=\left\{f\left(x_{0}\right)\right\}$. If $E$ is a peak set of $A$ containing $x_{0}$, then $h_{0}$ can be chosen so that $E\left(h_{0}\right) \subset E$. 
Observe that if $A$ is a function algebra on a metric space, then there are also peak functions $h \in \mathcal{P}_{x_{0}}^{0}(A)$ of $A$ that satisfy the conclusion of Proposition 1. This is true also for some non-function algebras, as the algebra of all Lipschitz functions on a metric space (e.g. 7]).

First we relax the somewhat restrictive peripherally multiplicative condition $\sigma_{\pi}(T f T g)=\sigma_{\pi}(f g)$ and assume, instead, that the peripheral spectra $\sigma_{\pi}(T f T g)$ do not diverge 'too much' from the peripheral spectra $\sigma_{\pi}(f g)$.

Theorem 1. Let $A \subset C(X)$ and $B \subset C(Y)$ be function algebras with $\delta A \subset X$ and $\delta B \subset Y$. If a surjective map $T: A \rightarrow B$ satisfies

(i) $\sigma_{\pi}(T f T g) \subset \mathcal{O}_{\varepsilon\|f g\|}\left(\sigma_{\pi}(f g)\right)$ for some $\varepsilon, 0 \leq \varepsilon<2 / 3$ and

(ii) $\|T f T g\|=\|f g\|$

for all $f \in A$ and all $g \in A$ with $\|g\|=1$, then there is a homeomorphism $\psi: \delta B \rightarrow$ $\delta A$ and a continuous function $\alpha: \delta B \rightarrow\{ \pm 1\}$ such that

$$
|(T f)(y)-\alpha(y) f(\psi(y))| \leq 2 \varepsilon|f(\psi(y))|
$$

for each $f \in A$ and every $y \in \delta B$. Consequently, $T$ is an almost weighted composition operator on $\delta B$.

Observe that if $T$ is norm-multiplicative, then both peripheral spectra, $\sigma_{\pi}(T f T g)$ and $\sigma_{\pi}(f g)$, are subsets of the circle $|z|=\|f g\|$. Hence, under the hypotheses of Theorem [1, the inclusion $\sigma_{\pi}(T f T g) \subset \mathcal{O}_{\varepsilon\|f g\|}\left(\sigma_{\pi}(f g)\right)$ holds automatically for any $\varepsilon \geq 2$.

Proof. Note that $p(A)=\delta A$ and $p(B)=\delta B$ since $A$ and $B$ are function algebras. As mentioned at the beginning of the section, the equality $(i i)$ implies that $T$ is a composition operator in modulus; i.e. there is a homeomorphism $\psi: \delta B \rightarrow \delta A$ so that $|(T f)(y)|=|f(\psi(y))|$ for all $f \in A$ and $y \in \delta B$.

Let $y_{0} \in \delta B, x_{0}=\psi\left(y_{0}\right) \in \delta A$ and let $f \in A$. If $f\left(x_{0}\right)=0$, then $\left|(T f)\left(y_{0}\right)\right|=$ $\left|f\left(x_{0}\right)\right|=0$, which implies (6). Suppose that $f\left(x_{0}\right) \neq 0$. Condition $(i)$ implies that $\left|T h_{1} T h_{2}-1\right| \leq \varepsilon$ for any pair $h_{1}, h_{2} \in \mathcal{P}_{x_{0}}(A)$ and all $x \in \delta A$. Hence Lemma 2 applies, and therefore there is a continuous function $\alpha: \delta B \rightarrow\{ \pm 1\}$ so that $\left|(T h)\left(y_{0}\right)-\alpha\left(y_{0}\right)\right| \leq \varepsilon$ for all $h \in \mathcal{P}_{\psi\left(y_{0}\right)}(A)$. By the Strong Bishop's Lemma (Proposition 1) there is a peaking function $h \in \mathcal{P}_{x_{0}}(A)$ such that $\sigma_{\pi}(f h)=\left\{f\left(x_{0}\right)\right\}$. Hence $x_{0} \in E(f h)$, and therefore $y_{0} \in E(T f T h)$, since $|(T f)(y)|=|f(\psi(y))|$ and $|(T h)(y)|=|h(\psi(y))|$ for all $y \in \delta B$. Consequently, $(T f T h)\left(y_{0}\right) \in \sigma_{\pi}(T f T h)$ and, by Lemmas 1 and 2 .

$$
\begin{gathered}
\left|(T f)\left(y_{0}\right)-\alpha\left(y_{0}\right) f\left(x_{0}\right)\right| \\
\leq\left|(T f)\left(y_{0}\right)-\alpha\left(y_{0}\right)(T f T h)\left(y_{0}\right)\right|+\left|\alpha\left(y_{0}\right)(T f T h)\left(y_{0}\right)-\alpha\left(y_{0}\right) f\left(x_{0}\right)\right| \\
=\left|(T f)\left(y_{0}\right)\right|\left|\alpha\left(y_{0}\right)(T h)\left(y_{0}\right)-1\right|+\mid(T f T h)\left(y_{0}\right)-f\left(\psi ( y _ { 0 } ) | \leq 2 \varepsilon | f \left(\psi\left(y_{0}\right) \mid,\right.\right.
\end{gathered}
$$

as desired.

Observe that the 'error' constant, $2 \varepsilon$, in the estimate (6) of the divergence between $T$ and $\alpha(f \circ \psi)$ is twice as big as the 'error' constant, $\varepsilon$, in the divergence between $\sigma_{\pi}(T f T g)$ and $\sigma_{\pi}(f g)$ under condition $(i)$.

In general, almost weighted composition operators are not necessarily weighted composition operators. Indeed, let $X$ be a compact Hausdorff space and let $A=$ $C(X)$. Consider the function $\kappa(f, x)=\exp \{i \varepsilon \operatorname{Re}(f(x) /\|f\|)\}$, where $f \in A$ and $x \in \delta A$. It is clear that $|\kappa(f, x)|=1$ and that for 'small' $\varepsilon$ 's, $|\kappa(f, x)-1|$ is also 
'small' for all $x \in \delta A$ and $f \in A$. Since $|\kappa(f, x) f(x)-f(x)| \leq|\kappa(f, x)-1||f(x)|$, the map $S: A \rightarrow A:(S f)(x)=\kappa(f, x) f(x)$ is an almost composition operator on $X$. However, $S$ is not a weighted composition operator, since, besides $x, \kappa$ depends also on $f$. Note that the operator $S$ is also norm-multiplicative.

Observe that if $f(\psi(y)) \neq 0$, then the inequality (6) can be written as

$$
\left|\frac{(T f)(y)}{\alpha(y) f(\psi(y))}-1\right| \leq 2 \varepsilon \text {. }
$$

Theorem 1 holds also if, instead of $(i), T$ satisfies its symmetric condition $\sigma_{\pi}(f g) \subset \mathcal{O}_{\varepsilon\|f g\|}\left(\sigma_{\pi}(T f T g)\right)$. It holds also for some non-uniformly closed algebras, provided that for every $f \in A$ and $x_{0} \in \delta A$ there is a peaking function $h \in \mathcal{P}_{x_{0}}(A)$ so that $\sigma_{\pi}(f h)=\left\{f\left(x_{0}\right)\right\}$ and $E(f h)=E(h)$, as is the case with the algebra of all Lipschitz functions on a metric space. In fact, as we shall see later (see Theorem 5 below), for such algebras much stronger results hold.

Since the condition $\sigma_{\pi}(T f T g) \subset \mathcal{O}_{\varepsilon\|f g\|}\left(\sigma_{\pi}(f g)\right.$ becomes $\sigma_{\pi}(T f T g) \subset \sigma_{\pi}(f g)$ when $\varepsilon=0$, Theorem 1 yields the main results in [3,5, 9, 12. Namely,

Corollary 1 ([10]). Let $X$ be a first countable locally compact Hausdorff space. If a surjective map $T: C(X) \rightarrow C(X)$ satisfies $\sigma(T f T g)=\sigma(f g), f, g \in A$, then there is a homeomorphism $\psi: \delta B \rightarrow \delta A$ and a continuous function $\alpha: \delta B \rightarrow\{ \pm 1\}$ so that $(T f)(y)=\alpha(y) f(\psi(y)), f \in A, y \in \delta B$; hence $T$ is a weighted composition operator on $\delta B$.

Corollary 2 (11, 12]). Let $A$ be a uniform or a function algebra. If a surjective map $T: A \rightarrow A$ satisfies $\sigma(T f T g)=\sigma(f g), f, g \in A$, then there is a homeomorphism $\psi: \delta B \rightarrow \delta A$ and a continuous function $\alpha: \delta B \rightarrow\{ \pm 1\}$ so that $(T f)(y)=\alpha(y) f(\psi(y)), f \in A, y \in \delta B$; thus $T$ is a weighted composition operator on $\delta B$.

Corollary 3 ([3]). Let $A$ and $B$ be uniform algebras. If a surjective map $T: A \rightarrow B$ satisfies $\operatorname{Ran}(T f T g)=\operatorname{Ran}(f g)$, where $\operatorname{Ran}(f)$ is the range of $f \in A$, then there is a homeomorphism $\psi: \delta B \rightarrow \delta A$ and a continuous function $\alpha: \delta B \rightarrow\{ \pm 1\}$ so that $(T f)(y)=\alpha(y) f(\psi(y)), f \in A, y \in \delta B$; therefore, $T$ is a weighted composition operator on $\delta B$.

Corollary 4 (9]). If a surjective map $T: A \rightarrow B$ between two uniform algebras satisfies $\sigma_{\pi}(T f T g)=\sigma_{\pi}(f g), f, g \in A$, then there is a homeomorphism $\psi: \delta B \rightarrow$ $\delta A$ and a continuous function $\alpha: \delta B \rightarrow\{ \pm 1\}$ so that $(T f)(y)=\alpha(y) f(\psi(y))$, $f \in A, y \in \delta B$; thus $T$ is a weighted composition operator on $\delta B$.

Corollary 5 ([5]). If a surjective map $T: A \rightarrow B$ between two function algebras satisfies $\sigma_{\pi}(T f T g) \subset \sigma_{\pi}(f g)$ (or, $\left.\sigma_{\pi}(f g) \subset \sigma_{\pi}(T f T g)\right), f, g \in A$, then there is a homeomorphism $\psi: \delta B \rightarrow \delta A$ and a continuous function $\alpha: \delta B \rightarrow\{ \pm 1\}$ so that $(T f)(y)=\alpha(y) f(\psi(y)), f \in A, y \in \delta B$; hence $T$ is a weighted composition operator.

Moreover, if in addition to the assumptions of Theorem 1 the peripheral spectra $\sigma_{\pi}(T f)$ are not 'too far' from the peripheral spectra $\sigma_{\pi}(f)$, then the map $T$ is an almost composition operator. Namely,

Theorem 2. Let $T: A \rightarrow B$ satisfy the hypotheses of Theorem 1. If there is a $\eta, 0 \leq \eta<1$, so that

$$
d\left(\sigma_{\pi}(T g), \sigma_{\pi}(g)\right) \leq \eta
$$


for all $f \in A$ and all $g \in A$ with $\|g\|=1$, then there is a homeomorphism $\psi: \delta B \rightarrow$ $\delta$ A so that

$$
|(T f)(y)-f(\psi(y))| \leq 2 \varepsilon|f(\psi(y))|
$$

for each $f \in A$ and every $y \in \delta B$. Therefore, $T$ is an almost composition operator on $\delta B$.

Proof. Let $\psi: \delta B \rightarrow \delta A, y_{0} \in \delta B$, and $x_{0}=\psi\left(y_{0}\right) \in \delta A$ be as in the proof of Theorem 1. If $V \subset \delta B$ is a neighborhood of $y_{0}$, denote $U=\psi(V)$. Hence $x_{0} \in U$, and there is a peaking function $h \in \mathcal{P}_{x_{0}}(B)$ with $E(h) \subset U$, since $x_{0}$ is a $p$-point of $A$. By condition (7) there is a $y_{1} \in \delta B$ with $(T h)\left(y_{1}\right) \in \sigma_{\pi}\left(T h_{1}\right)$ such that

$$
\left|(T h)\left(y_{1}\right)-1\right|=d\left((T h)\left(y_{1}\right), 1\right)=d\left(\sigma_{\pi}(T h), \sigma_{\pi}(h)\right) \leq \eta .
$$

By Lemma 2 there is a continuous function $\alpha: \delta B \rightarrow\{ \pm 1\}$ with $|(T h)(y)-\alpha(y)| \leq \varepsilon$ for all $y \in \delta B$. Therefore,

$$
\left|\alpha\left(y_{1}\right)-1\right| \leq\left|\alpha\left(y_{1}\right)-(T h)\left(y_{1}\right)\right|+\left|(T h)\left(y_{1}\right)-1\right| \leq \varepsilon+\eta<5 / 3 .
$$

Hence for any neighborhood $V$ of $y_{0}$ there is a point $y_{1} \in V$ with $\left|\alpha\left(y_{1}\right)-1\right| \leq 5 / 3$. The continuity of $T h$ at $y_{0}$ implies $\left|\alpha\left(y_{0}\right)-1\right| \leq 5 / 3<2$; thus $\alpha\left(y_{0}\right)=1$, since $\alpha= \pm 1$. Consequently, $\alpha \equiv 1$ and the result follows from Theorem 1 .

Note that the 'error' constant, $2 \varepsilon$, in the estimate (8) of the divergence between $T$ and $f \circ \psi$ does not depend on the distance, $\eta<1$, between $\sigma_{\pi}(T g)$ and $\sigma_{\pi}(g)$.

In [5] it is shown that if $T: A \rightarrow B$ is a surjection between function algebras such that $\sigma_{\pi}(T f T g) \subset \sigma_{\pi}(f g)$ (or, $\sigma_{\pi}(f g) \subset \sigma_{\pi}(T f T g)$ ), then $T$ is a weighted composition operator. As a corollary from Theorem 2 we obtain that if, in addition, the peripheral spectra $\sigma_{\pi}(T f)$ are not 'too far' from the peripheral spectra $\sigma_{\pi}(f)$, then $T$ is actually a composition operator on $\delta B$, which generalizes and extends the main results in [5] and [9].

Theorem 3. If a surjection $T: A \rightarrow B$ between function algebras satisfies

(i) $\sigma_{\pi}(T f T g) \subset \sigma_{\pi}(f g)\left(\right.$ or $\left.\sigma_{\pi}(f g) \subset \sigma_{\pi}(T f T g)\right)$ and

(ii) $d\left(\sigma_{\pi}(T g), \sigma_{\pi}(g)\right) \leq \eta$ for some $\eta, 0 \leq \eta<1$,

for all $f \in A$ and all $g \in A$ with $\|g\|=1$, then there is a homeomorphism $\psi: \delta B \rightarrow$ $\delta A$ so that

$$
(T f)(y)=f(\psi(y))
$$

for each $f \in A$ and every $y \in \delta B$. Consequently, $T$ is a composition operator on $\delta B$, and therefore an algebra isomorphism.

Indeed, in this case, Theorem 2 holds with $\varepsilon=0$.

Corollary 6. If a surjection $T: A \rightarrow B$ between function algebras satisfies

(i) $\sigma_{\pi}(T f T g) \subset \mathcal{O}_{\varepsilon\|f g\|}\left(\sigma_{\pi}(f g)\right)\left(\right.$ or $\left.\sigma_{\pi}(f g) \subset \mathcal{O}_{\varepsilon\|f g\|}\left(\sigma_{\pi}(T f T g)\right)\right)$ for some $\varepsilon, 0 \leq \varepsilon<2 / 3$,

(ii) $\sigma_{\pi}(T g) \cap \sigma_{\pi}(g) \neq \varnothing$, and

(iii) $\|T f T g\|=\|f g\|$

for all $f \in A$ and all $g \in A$ with $\|g\|=1$, then there is a homeomorphism $\psi: \delta B \rightarrow$ $\delta A$ so that

$$
|(T f)(y)-f(\psi(y))| \leq 2 \varepsilon|f(\psi(y))|
$$

for each $f \in A$ and every $y \in \delta B$. Thus $T$ is an almost composition operator on $\delta B$, and therefore an almost isomorphism between algebras. 
In this case Theorem 2 holds with $\eta=0$.

Corollary 7. If a surjection $T: A \rightarrow B$ between function algebras satisfies

(i) $\sigma_{\pi}(T f T g) \subset \mathcal{O}_{\varepsilon\|f g\|}\left(\sigma_{\pi}(f g)\right)\left(\right.$ or $\left.\sigma_{\pi}(f g) \subset \mathcal{O}_{\varepsilon\|f g\|}\left(\sigma_{\pi}(T f T g)\right)\right)$ for some $\varepsilon, 0 \leq \varepsilon<2 / 3$,

(ii) $\sigma_{\pi}(T g) \subset \mathcal{O}_{\eta}\left(\sigma_{\pi}(g)\right)$ (or $\left.\sigma_{\pi}(g) \subset \mathcal{O}_{\eta}\left(\sigma_{\pi}(T g)\right)\right)$ for some $\eta, 0 \leq \eta<1$, and (iii) $\|T f T g\|=\|f g\|$

for all $f \in A$ and all $g \in A$ with $\|g\|=1$, then there is a homeomorphism $\psi: \delta B \rightarrow$ $\delta A$ so that

$$
|(T f)(y)-f(\psi(y))| \leq 2 \varepsilon|f(\psi(y))|
$$

for each $f \in A$ and every $y \in \delta B$. Hence $T$ is an almost composition operator on $\delta B$ and, therefore, an almost isomorphism between algebras.

Indeed, condition (ii) implies that $d\left(\sigma_{\pi}(T g), \sigma_{\pi}(g)\right) \leq \eta$ for every $g \in A$ with $\|g\|=1$, and the result follows from Theorem 2. Here the 'error' constant, $2 \varepsilon$, in the estimate of the divergence between $T$ and $\alpha(f \circ \psi)$ does not depend on the 'error' constant, $\eta$, in the divergence between $\sigma_{\pi}(T f)$ and $\sigma_{\pi}(f)$ under condition (ii).

Below we also relax the weak peripherally multiplicative condition $\sigma_{\pi}(T f T g) \cap$ $\sigma_{\pi}(f g) \neq \varnothing$ and assume, instead, that the peripheral spectra $\sigma_{\pi}(T f T g)$ are not 'too far' from the peripheral spectra $\sigma_{\pi}(f g)$. Namely,

Theorem 4. Let $A \subset C(X), B \subset C(Y)$ be function algebras with $\delta A \subset X$ and $\delta B \subset Y$. If a surjective map $T: A \rightarrow B$ satisfies

(i) $d\left(\sigma_{\pi}(T f T g), \sigma_{\pi}(f g)\right) \leq \varepsilon\|f g\|$ for some $\varepsilon, 0 \leq \varepsilon<1$,

(ii) $\sigma_{\pi}(T g) \subset \mathcal{O}_{\eta}\left(\sigma_{\pi}(g)\right)\left(\right.$ or $\left.\sigma_{\pi}(g) \subset \mathcal{O}_{\eta}\left(\sigma_{\pi}(T g)\right)\right)$ for some $\eta, 0 \leq \eta<1$, and

(iii) $\|T f T g\|=\|f g\|$

for all $f \in A$ and all $g \in A$ with $\|g\|=1$, then there is a homeomorphism $\psi: \delta B \rightarrow$ $\delta A$ so that

$$
|(T f)(y)-f(\psi(y))| \leq(\varepsilon+\eta)|f(\psi(y))|
$$

for each $f \in A$ and every $y \in \delta B$. Therefore, $T$ is an almost composition operator on $\delta B$, hence, an almost isomorphism between algebras.

Proof. Let, as before, $\psi: \delta B \rightarrow \delta A$ be the homeomorphism mentioned at the beginning of the section such that $|(T f)(y)|=|f(\psi(y))|$ for all $y \in \delta B$ and $f \in A$. Let $y_{0} \in \delta B, x_{0}=\psi\left(y_{0}\right) \in \delta A$, and let $f \in A$ be such that $f\left(x_{0}\right) \neq 0$. Let $V \subset \delta B$ be an open neighborhood of $y_{0}$ and $U=\psi(V)$. Clearly, $x_{0}=\psi^{-1}\left(y_{0}\right) \in U$. By the Strong Bishop's Lemma (Proposition 1) there is a peaking function $h \in \mathcal{P}_{x_{0}}(A)$ with $E(f h)=E(h) \subset U$ such that $\sigma_{\pi}(f h)=\left\{f\left(x_{0}\right)\right\}$. Since $|(T f)(y)|=|f(\psi(y))|$, $f \in A, y \in \delta B$, we have $E(T f T h)=E(T h) \subset V$. Condition $(i)$ implies that there is an $y_{1} \in E(T f T h) \subset V$ so that

$$
\left|(T f T h)\left(y_{1}\right)-f\left(x_{0}\right)\right|=d\left((T f T h)\left(y_{1}\right), f\left(x_{0}\right)\right) \leq \varepsilon\|f h\|=\varepsilon\left|f\left(x_{0}\right)\right| .
$$

Since $\sigma_{\pi}(h)=\{1\}$ and $(T h)\left(y_{1}\right) \in \sigma_{\pi}(T h)$, condition $(i i)$ yields $\left|(T h)\left(y_{1}\right)-1\right| \leq \eta$, and therefore

$$
\begin{gathered}
\left|(T f)\left(y_{1}\right)-f\left(x_{0}\right)\right| \\
\leq\left|(T f)\left(y_{1}\right)-(T f T h)\left(y_{1}\right)\right|+\left|(T f T h)\left(y_{1}\right)-f\left(x_{0}\right)\right| \leq \eta\left|(T f)\left(y_{1}\right)\right|+\varepsilon\left|f\left(x_{0}\right)\right| .
\end{gathered}
$$


Hence, for any neighborhood $V$ of $y_{0}$ there is a $y_{1} \in V$ with $\left|(T f)\left(y_{1}\right)-f\left(x_{0}\right)\right| \leq$ $\varepsilon\left|f\left(x_{0}\right)\right|+\eta\left|(T f)\left(y_{1}\right)\right|$. The continuity of $T f$ at $y_{0}$ implies $\left|(T f)\left(y_{0}\right)-f\left(x_{0}\right)\right| \leq$ $\varepsilon\left|f\left(x_{0}\right)\right|+\eta\left|(T f)\left(y_{0}\right)\right|=(\varepsilon+\eta)\left|f\left(\psi\left(y_{0}\right)\right)\right|$, as claimed.

Observe that the 'error' constant, $\varepsilon+\eta$, in the estimate (10) of the divergence between $T$ and $\alpha(f \circ \psi)$ depends on the 'error' constant, $\varepsilon$, in the proximity between $\sigma_{\pi}(T f T g)$ and $\sigma_{\pi}(f g)$ under condition $(i)$, and also on the divergence, $\eta$, between $\sigma_{\pi}(T f)$ and $\sigma_{\pi}(f)$ under condition $(i i)$.

Since, when $\varepsilon=0$, the assumption $d\left(\sigma_{\pi}(T f T g), \sigma_{\pi}(f g)\right) \leq \varepsilon\|f g\|$ becomes $\sigma_{\pi}(T f T g) \cap \sigma_{\pi}(f g) \neq \varnothing$, as a corollary, we obtain the following immediate generalization of the main result in [5], 8] and [14].

Proposition 2. If a surjection $T: A \rightarrow B$ between function algebras satisfies

(i) $\sigma_{\pi}(T f T g) \cap \sigma_{\pi}(f g) \neq \varnothing$ and

(ii) $\sigma_{\pi}(T g) \subset \mathcal{O}_{\eta}\left(\sigma_{\pi}(g)\right)$ (or $\sigma_{\pi}(g) \subset \mathcal{O}_{\eta}\left(\sigma_{\pi}(T g)\right)$ ) for some $\eta, 0 \leq \eta<2$, for all $f \in A$ and all $g \in A$ with $\|g\|=1$, then

$$
|(T f)(y)-f(\psi(y))| \leq \eta|f(\psi(y))|
$$

for each $y \in \delta B$ and every $f \in A$. Hence $T$ is an almost composition operator on $\delta B$, therefore, an almost isomorphism between algebras.

The main results in [5, 14] are direct consequences of Proposition 2, Namely,

Corollary 8 ([14]). If a surjection $T: A \rightarrow B$ between function algebras satisfies $\left.\sigma_{\pi}(T f T g) \cap \sigma_{\pi}(f g)\right) \neq \varnothing$ and $\sigma_{\pi}(T g)=\sigma_{\pi}(g)$ for all $f, g \in A$, then there is a homeomorphism $\psi: \delta B \rightarrow \delta A$ so that $(T f)(y)=f(\psi(y)), y \in \delta B, f \in A$; thus $T$ is a composition operator on $\delta B$.

Corollary 9 ([5]). If a surjection $T: A \rightarrow B$ between function algebras satisfies $\left.\sigma_{\pi}(T f T g) \cap \sigma_{\pi}(f g)\right) \neq \varnothing$ and $\sigma_{\pi}(T g) \subset \sigma_{\pi}(g)\left(\right.$ or $\left.\sigma_{\pi}(g) \subset \sigma_{\pi}(T g)\right)$ for all $f, g \in A$, then there is a homeomorphism $\psi: \delta B \rightarrow \delta A$ so that $(T f)(y)=f(\psi(y)), y \in \delta B$, $f \in A$; hence, $T$ is a composition operator on $\delta B$.

Indeed, in both of these cases Theorem 4 holds with $\varepsilon=\eta=0$.

Note that the uniform closedness of the algebras $A$ or $B$ in the previous results is needed only to make sure that the Strong Bishop's Lemma (Proposition 1) applies. The results are true also for any non-uniformly closed algebras of functions, for which the conclusion of Proposition 1 holds.

In the case when for every $f \in A$ and each $x \in \delta A$ there is a peak (not just peaking) function $h \in \mathcal{P}_{x}^{0}(A)$ with $\sigma_{\pi}(f h)=\{f(x)\}$, condition (ii) in Theorem 4 is, in fact, unnecessary, and we have the following more general result.

Theorem 5. Let $A$ and $B$ be algebras of bounded continuous functions on locally compact Hausdorff spaces $X$ and $Y$ with $p(A)=\delta A \subset X$ and $p(B)=\delta B \subset Y$. Assume that for every $f \in A$ and each $x \in \delta A$ there is a peak function $h \in \mathcal{P}_{x}^{0}(A)$ with $\sigma_{\pi}(f h)=\{f(x)\}$. If a surjective map $T: A \rightarrow B$ satisfies

(i) $d\left(\sigma_{\pi}(T f T g), \sigma_{\pi}(f g)\right) \leq \varepsilon\|f g\|$ for some $\varepsilon, 0 \leq \varepsilon<2 / 3$ and

(ii) $\|T f T g\|=\|f g\|$

for all $f \in A$ and all $g \in A$ with $\|g\|=1$, then there is a homeomorphism $\psi: \delta B \rightarrow$ $\delta A$ and a continuous function $\alpha: A \rightarrow\{ \pm 1\}$ such that

$$
|(T f)(y)-\alpha(y) f(\psi(y))| \leq 2 \varepsilon|f(\psi(y))|
$$


for each $f \in A$ and every $y \in \delta B$. Consequently, $T$ is an almost weighted composition operator on $\delta B$.

Proof. Since $T$ is norm-multiplicative, then, as before, there is a homeomorphism $\psi: \delta B \rightarrow \delta A$ so that $|(T f)(y)|=\mid f(\psi(y) \mid=1$ for all $y \in \delta B$ and $f \in A$. If $h_{1}, h_{2} \in \mathcal{P}_{\psi(y)}^{0}(A)$, then $E\left(h_{1} h_{2}\right)=\{\psi(y)\}$ and $\sigma_{\pi}\left(h_{1} h_{2}\right)=\{1\}$. Therefore, $E\left(T h_{1} T h_{2}\right)=\{y\}$, and thus $\sigma_{\pi}\left(T h_{1} T h_{2}\right)=\left\{\left(T h_{1} T h_{2}\right)(y)\right\}$. Condition $(i)$ implies that $\left|\left(T h_{1} T h_{2}\right)(y)-1\right| \leq \varepsilon$. Hence, $\sigma_{\pi}\left(T h_{1} T h_{2}\right)=\left\{\left(T h_{1} T h_{2}\right)(y)\right\} \subset \mathcal{O}_{\varepsilon}(1)$. By Lemma 2 there is a continuous function $\alpha: \delta B \rightarrow\{ \pm 1\}$ such that $|(T h)(y)-\alpha(y)| \leq$ $\varepsilon$ for all $y \in \delta B$ and $h \in \mathcal{P}_{\psi(y)}^{0}(A)$.

Let $y_{0} \in \delta B, x_{0}=\psi\left(y_{0}\right) \in \delta A$, and let $f \in A$ be such that $f\left(x_{0}\right) \neq 0$. Assume that $\sigma_{\pi}(f h)=\left\{f\left(x_{0}\right)\right\}$ for some $h \in \mathcal{P}_{\psi(y)}^{0}(A)$. By Lemma 1,

$$
|(T f T h)(y)-f(\psi(y))| \leq \varepsilon|f(\psi(y))|
$$

for each $y \in \delta B$ and every $f \in A$, and therefore

$$
\begin{gathered}
\left|(T f)\left(y_{0}\right)-\alpha\left(y_{0}\right) f\left(x_{0}\right)\right| \\
\leq\left|(T f)\left(y_{0}\right)-\alpha\left(y_{0}\right)(T f T h)\left(y_{0}\right)\right|+\left|\alpha\left(y_{0}\right)(T f T h)\left(y_{0}\right)-\alpha\left(y_{0}\right) f\left(x_{0}\right)\right| \\
\leq\left|(T f)\left(y_{0}\right)\right|\left|\alpha\left(y_{0}\right)(T h)\left(y_{0}\right)-1\right|+\left|(T f T h)\left(y_{0}\right)-f\left(x_{0}\right)\right| \leq 2 \varepsilon\left|f\left(\psi\left(y_{0}\right)\right)\right|,
\end{gathered}
$$

as desired.

Note that here the 'error' constant, $2 \varepsilon$, in the estimate of the divergence between $T$ and $\alpha(f \circ \psi)$ is twice as big as the 'error' constant, $\varepsilon$, in the proximity between $\sigma_{\pi}(T f T g)$ and $\sigma_{\pi}(f g)$ under condition $(i)$.

Algebras satisfying the conditions of Theorem 5 are, for instance, function algebras on metric spaces or the algebra of all Lipschitz functions on a metric space.

Since, when $\varepsilon=0$, the assumption $d\left(\sigma_{\pi}(T f T g), \sigma_{\pi}(f g)\right) \leq \varepsilon\|f g\|$ becomes $\sigma_{\pi}(T f T g) \cap \sigma_{\pi}(f g) \neq \varnothing$, Theorem [5 yields one of the main results in [6], and, together, its special cases in [7,13]:

Corollary $10([\underline{6})$. Let $A$ and $B$ be dense subalgebras of function algebras on $X$ and $Y$ with $p(A)=\delta A \subset X$ and $p(B)=\delta B \subset Y$. Assume that for every $f \in A$ and each $x \in \delta A$ there is a peak function $h \in \mathcal{P}_{x}^{0}(A)$ with $\sigma_{\pi}(f h)=\{f(x)\}$. If a surjective map $T: A \rightarrow B$ satisfies $\sigma_{\pi}(T f T g) \cap \sigma_{\pi}(f g) \neq \varnothing$ for all $f, g \in A$, then $(T f)(y)=\alpha(y) f(\psi(y)), f \in A, y \in \delta B$, where $\alpha: \delta B \rightarrow\{ \pm 1\}$ is a continuous function, i.e. $T$ is a weighted composition operator.

If, in addition to the hypotheses of Theorem 5, there is an $\eta, 0 \leq \eta<1$, so that $d\left(\sigma_{\pi}(T g), \sigma_{\pi}(g)\right) \leq \eta$ for all $f \in A$ and $g \in A$ with $\|g\|=1$, then

$$
|(T f)(y)-f(\psi(y))| \leq 2 \varepsilon|f(\psi(y))|
$$

for all $f \in A$ and $y \in \delta B$. Thus $T$ is an almost composition operator, and therefore an almost isomorphism between algebras. Indeed, under the hypotheses, in addition to the inequality $|(T h)(y)-\alpha(y)| \leq \varepsilon$ we also have $|(T h)(y)-1| \leq \eta<1$ for any $h \in \mathcal{P}_{\psi(y)}^{0}(A)$. Therefore,

$$
|\alpha(y)-1| \leq|\alpha(y)-(T h)(y)|+|(T h)(y)-1| \leq \varepsilon+\eta<2 .
$$

Consequently, the function $\alpha$ in Theorem 5 is identically equal to one. In this case the 'error' constant, $2 \varepsilon$, in the estimate of the divergence between $T$ and $f \circ \psi$ does not depend on the proximity, $\eta<1$, between $\sigma_{\pi}(T g)$ and $\sigma_{\pi}(g)$. 


\section{ACKNOWLEDGMENT}

Thanks are due to the referees for their instructive and helpful comments and suggestions.

\section{REFERENCES}

[1] S. A. Grigoryan and T. V. Tonev, Shift-invariant uniform algebras on groups, Instytut Matematyczny Polskiej Akademii Nauk. Monografie Matematyczne (New Series) [Mathematics Institute of the Polish Academy of Sciences. Mathematical Monographs (New Series)], vol. 68, Birkhäuser Verlag, Basel, 2006. MR2239563 (2007m:46074)

[2] Osamu Hatori, Scott Lambert, Aaron Luttman, Takeshi Miura, Thomas Tonev, and Rebekah Yates, Spectral preservers in commutative Banach algebras, Function spaces in modern analysis, Contemp. Math., vol. 547, Amer. Math. Soc., Providence, RI, 2011, pp. 103-123, DOI 10.1090/conm/547/10812. MR2856485(2012j:46066)

[3] Osamu Hatori, Takeshi Miura, and Hiroyuki Takagi, Characterizations of isometric isomorphisms between uniform algebras via nonlinear range-preserving properties, Proc. Amer. Math. Soc. 134 (2006), no. 10, 2923-2930 (electronic), DOI 10.1090/S0002-9939-06-08500-5. MR2231616(2007h:46063)

[4] Krzysztof Jarosz, Perturbations of Banach algebras, Lecture Notes in Mathematics, vol. 1120, Springer-Verlag, Berlin, 1985. MR788884 (86k:46074)

[5] J. Johnson and T. Tonev, Spectral conditions for weighted composition operators on function algebras, Communications in Math. and Appl. 3 (2012), 51-59.

[6] Jeffrey Johnson, Peripherally-multiplicative spectral preservers between function algebras, Ph.D. thesis, University of Montana, 2013.

[7] Kristopher Lee and Aaron Luttman, Generalizations of weakly peripherally multiplicative maps between uniform algebras, J. Math. Anal. Appl. 375 (2011), no. 1, 108-117, DOI 10.1016/j.jmaa.2010.08.051. MR2735698 (2012c:46118)

[8] Scott Lambert, Aaron Luttman, and Thomas Tonev, Weakly peripherally-multiplicative mappings between uniform algebras, Function spaces, Contemp. Math., vol. 435, Amer. Math. Soc., Providence, RI, 2007, pp. 265-281, DOI 10.1090/conm/435/08383. MR2359435 (2009b:46108)

[9] Aaron Luttman and Thomas Tonev, Uniform algebra isomorphisms and peripheral multiplicativity, Proc. Amer. Math. Soc. 135 (2007), no. 11, 3589-3598 (electronic), DOI 10.1090/S0002-9939-07-08881-8. MR2336574 (2008k:46150)

[10] Lajos Molnár, Some characterizations of the automorphisms of $B(H)$ and $C(X)$, Proc. Amer. Math. Soc. 130 (2002), no. 1, 111-120 (electronic), DOI 10.1090/S0002-9939-01-06172-X. MR.1855627 (2002m:47047)

[11] N. V. Rao and A. K. Roy, Multiplicatively spectrum-preserving maps of function algebras, Proc. Amer. Math. Soc. 133 (2005), no. 4, 1135-1142 (electronic), DOI 10.1090/S0002-993904-07615-4. MR2117215(2005k:46124)

[12] N. V. Rao and A. K. Roy, Multiplicatively spectrum-preserving maps of function algebras. II, Proc. Edinb. Math. Soc. (2) 48 (2005), no. 1, 219-229, DOI 10.1017/S0013091504000719. MR2117721(2005k:46125)

[13] Rumi Shindo, Weakly-peripherally multiplicative conditions and isomorphisms between uniform algebras, Publ. Math. Debrecen 78 (2011), no. 3-4, 675-685, DOI 10.5486/PMD.2011.4937. MR2867209 (2012j:46073)

[14] Thomas Tonev, Weak multiplicative operators on function algebras without units, Banach algebras 2009, Banach Center Publ., vol. 91, Polish Acad. Sci. Inst. Math., Warsaw, 2010, pp. 411-421, DOI 10.4064/bc91-0-24. MR2777467(2012f:46090)

Department of Mathematics, University of Montana, Missoula, Montana 59812

E-mail address: tonevtv@mso.umt.edu 